\title{
Analysis of factors correlated with spinal clinically isolated syndrome conversion to multiple sclerosis
}

\author{
Jian $\mathrm{Li}^{1}$, Qing-Li Sun ${ }^{1}$, Yi-Ming Zhao ${ }^{2}$ \\ ${ }^{1}$ Department of Neurology, Peking University Third Hospital, Beijing, China \\ ${ }^{2}$ Centre for Clinical Epidemiology, Peking University Third Hospital, Beijing, China
}

\begin{abstract}
Introduction. The present study aims to explore the factors influencing spinal clinically isolated syndrome (CIS) conversion to multiple sclerosis (MS).

Material and methods. Sixty-one patients diagnosed with spinal CIS from January 2010 to November 2020 were divided into a non-progressing (CIS) group with 27 patients, and a conversion to MS (MS) group with 34 patients, based on whether they had converted to MS. The clinical presentation at onset, the Expanded Disability Status Scale (EDSS) before and after steroid therapy, the results of magnetic resonance imaging (MRI), the oligoclonal bands in cerebrospinal fluid (CSF-OCB), and the evoked potentials (EPs) were retrospectively analysed.

Results. Differences in gender and age were not statistically significant between the MS and CIS groups. The median time to relapse was 12 months for the MS group, with an upper quartile of 23.7 months, and $91.2 \%$ of patients relapsed within three years. In univariate analysis, patients with CIS beginning with sensory symptoms had a lower level of progression to MS $(\mathrm{OR}=0.311)$. Patients with Kurtzke Functional Systems Scores (FSSs) of pyramidal functions $\geq 2$ ( $\mathrm{OR}=3.582$ ) and positive CSF$-\mathrm{OCB}(\mathrm{OR}=5.208)$ quickly progressed to MS. There was no significant difference between the two groups in terms of spinal cord lesions $<3$ vertebral segments, gadolinium enhancing lesions, or abnormal EPs. The difference in the EDSS scores before and after steroid therapy was higher in the MS group than in the CIS group ( $p=0.001)$. Differences of $\geq 1.5$ in the EDSS scores before and after steroid therapy were risk factors for CIS conversion to MS (OR = 9.333).

Conclusions. Patients with spinal CIS with pure sensory abnormalities at onset were less likely to convert to MS ( $O R=0.311$ ), and the risk factors were, in order of risk, the difference in EDSS score before and after steroid therapy $(\geq 1.5 ; O R=9.333)$, positive CSF-OCB (OR = 5.208), and those with an FSS of the pyramidal functions score $\geq 2 ; O R=3.582)$. The present study serves as a simple 'first step'. Any potential predictors identified should be validated via future prospective studies.
\end{abstract}

Key words: spinal clinically isolated syndrome, multiple sclerosis, EDSS before and after steroid therapy, CSF-OCB

(Neurol Neurochir Pol 2022; 56 (4): 318-325)

\section{Introduction}

Clinically isolated syndromes (CISs) are demyelinating disorders of the central nervous system (CNS) that first develop as a solitary lesion and primarily involve unilateral optic neuritis, multifocal supratentorial syndrome, focal brainstem or cerebellar syndrome, and spinal CIS [1]. Some $30-70 \%$ of patients with CIS develop multiple sclerosis (MS) [2]. Early disease-modifying therapy (DMT) can benefit patients at a higher risk of progression to MS. Therefore, early prediction of the conversion of patients with CIS, and identification of risk factors for the advancement of CIS to MS, would be of significant value. Clinical studies have suggested that the confirmed potential predictors for the conversion of CIS patients to MS are an increased $(>10)$ baseline related to brain and spinal cord magnetic resonance imaging (MRI) T2-hyperintense lesions,

Address for correspondence: Jian Li, Department of Neurology, Peking University Third Hospital, No. 49 Huayuan North Road, Haidian District,

Beijing 100191, China; e-mail: LIJ698524@163.com

Received: 19.10.2021 Accepted: 23.12.2021 Early publication date: 14.02.2022

This article is available in open access under Creative Common Attribution-Non-Commercial-No Derivatives 4.0 International (CC BY-NC-ND 4.0) license, allowing to download articles and share them with others as long as they credit the authors and the publisher, but without permission to change them in any way or use them commercially. 
oligoclonal band (OCB) positivity, and subclinical evoked potential (EP) abnormalities [3]. Patients with three or more MRI lesions were more likely to develop MS than those with fewer than three lesions [4]. Optic nerve and spinal cord injuries are common in Chinese patients with MS. Patients tend to visit the ophthalmology department with the first episode of optic neuritis. The characteristics of patients presenting with spinal CIS on their first visit to the neurology department at our hospital were retrospectively analysed so as to explore high-risk factors for the conversion of spinal CIS to MS.

\section{Material and methods}

\section{General characteristics}

From January 2010 to November 2020, 61 patients with spinal cord CIS who had first been diagnosed at the neurology department of our hospital were divided into two groups according to whether the follow-up results turned out to be MS. There were 27 patients in the non-conversion (CIS) group, with 11 males and 16 females with an average age of 39.4 \pm 11.9 years. The converted to MS group included 34 patients, nine males and 25 females, and the average age was 38.4 \pm 11.2 years. The spinal CIS and MS diagnoses referred to the 2017 McDonald diagnostic criteria for MS [1]. The ethics committee of our hospital approved the present study (project number M2020513), and all participants signed an informed consent form for inclusion in this study.

\section{Inclusion and exclusion criteria}

The inclusion criteria were as follows: (1) patient aged $\geq 18$ years; (2) patient with a diagnosis of spinal CIS on the first visit, who either did or did not convert to MS later; (3) patient who did not convert to MS with a follow-up duration of more than 36 months. The exclusion criteria were as follows: (1) patient without a cranial and spinal cord MRI at the first episode, patient without cerebrospinal fluid (CSF) examination, and patient who did not complete the visual evoked potentials (VEPs) and brainstem auditory evoked potentials (BAEPs); (2) patient with other serious disease such as malignant tumour and hepatic/renal dysfunction; (3) pregnant and lactating females.

\section{Clinical data}

When the patient was first hospitalised, the symptoms at the onset of disease, clinical characteristics, and pre-treatment Expanded Disability Status Scale (EDSS) scores were recorded. All patients received high-dose methylprednisolone shock therapy at the first onset via an intravenous drip, with the dosage halved every three days until disuse as follows: $1 \mathrm{~g} / \mathrm{d}$, $0.5 \mathrm{~g} / \mathrm{d}, 0.24 \mathrm{~g} / \mathrm{d}, 0.12 \mathrm{~g} / \mathrm{d}$, and prednisone oral $80 \mathrm{mg} / \mathrm{d}$. This took $15.63 \pm 5.12$ days in the CIS group and $16.62 \pm 4.98$ days in the MS group $(t=0.761 ; \mathrm{p}=0.450)$. The time between this and the spinal MRI examination was $3.93 \pm 1.33$ days in the
CIS group and $4.24 \pm 1.48$ days in the MS group $(t=0.849$; $\mathrm{p}=0.399$ ) with no significant differences observed between the two groups. The EDSS score after two weeks of steroid therapy, the follow-up after discharge, the time to conversion in the MS group, and the duration of follow-up in the CIS group, were all recorded by two neurologists with neurostatus e-test qualifications.

\section{Image data}

Using a Siemens 3T MRI, two radiologists reviewed the radiographs and made imaging diagnoses, and $\mathrm{T} 1$-weighted images (T1WI), T2-weighted images (T2WI), and Gadolinium-diethylenetriamine pentaacetic acid (Gd-DTPA) enhancements of the spinal cord were scanned at the onset. The T1WI, T2WI, fluid-attenuated inversion recovery (FLAIR) of the brain MRI were scanned. The Gd-DTPA enhanced scanning of the brain MRI was performed in patients with intracranial lesions. The time from onset to the first spinal cord MRI examination was $11.74 \pm 4.29$ days in the CIS group and $12.38 \pm 4.25$ days in the MS group $(t=0.583$; $\mathrm{p}=0.562)$; the results showed no significant difference between the two groups.

\section{Neurophysiological data}

A Medtronic Functional Diagnostic Keypoint Workstation was used to conduct the evoked potential tests. The pattern-reversal visual evoked potential and BAEP tests were conducted at the onset of the disease. The VEP abnormalities were determined by the following: (1) latency $>$ mean +3 standard deviation (SD); (2) an amplitude $<3 \mu \mathrm{v}$ and poorly differentiated or absent waveforms; (3) a latency difference of P100 between the eyes greater than 8-10 ms. The criteria for abnormal BAEP were as follows: (1) the prolonged latency of each wave $>$ mean $+3 \mathrm{SD}$, prolonged wave interval $>$ mean $+3 \mathrm{SD}$; (2) the disappearance of waveform or amplitude (I/V) $>200 \%$; (3) (III-V) / (I-III > 1.0).

\section{Examination of CSF}

Isoelectric focusing electrophoresis and immunofixation staining were used to detect OCBs in the cerebrospinal fluid (CSF-OCB) and blood using the Sebia Hydrasys 2 system. The OCB results were divided into five types, i.e. type 1 , no bands in the serum and CSF; type 2, two or more bands in the CSF but none in the serum; type 3 , bands in both the serum and the CSF, but more bands in the CSF; type 4, serum and CSF had the same amount of bands, and the distribution in both was the same; type 5 , monoclonal bands in the serum and the CSF. Among these, types 1, 4, and 5 were negative for CSF-OCB, while types 2 and 3 were positive [5]. The concentrations of immunoglobulin $\mathrm{G}(\mathrm{IgG})$ and albumin in the CSF were determined by rocket immunoelectrophoresis; an $\operatorname{IgG}$ index $>0.7$ and an $\operatorname{IgG}$ synthesis rate $>10 \mathrm{mg} / 24 \mathrm{~h}$ were considered abnormal. 


\section{Other assays}

Other assays used in the evaluations were the serum autoimmune antibody assay and the serum and CSF aquaporin-4 antibodies (AQP4-Ab), as well as anti-myelin oligodendrocyte glycoprotein antibody (MOG-Ab). The AQP4-Ab was detected by the entire length of the AQP4 peptide being expressed in the recombinant human embryonic kidney 293 cell line, which was transfected using a EUROIMMUN indirect immunofluorescence kit and observed using a EUROStar III Plus fluorescence microscope. The MOG-Ab was determined using an enzyme-linked immunosorbent assay. The MOG antigen and antibody were Santa Cruz products, and the OD values were measured at $492 \mathrm{~nm}$ using $\sum 960$ micrometers.

\section{Statistical methods}

The SPSS Statistics 21.0 software was adopted for data analysis. The measurement data that satisfied normal distribution were expressed as mean $\pm \mathrm{SD}(\mathrm{X} \pm \mathrm{S})$; those that did not satisfy normal distribution were expressed as the median. A t-test was used for making comparisons between the two groups. The countable data was expressed as a percentage, and the chi-squared $(\chi 2)$ test was adopted for intergroup comparisons. The median was analysed by a rank-sum test. All data was tested using a two-sided method; $\mathrm{p}<0.05$ was considered statistically significant.

\section{Results}

\section{General characteristics}

The MS group included nine males and 25 females, while the CIS group had 11 males and 16 females; $55.7 \%$ of the patients returned to MS; the $\chi 2$ test result for the gender composition $=1.906$ with $\mathrm{p}=0.238$. The average age in the MS group was $38.44 \pm 11.23$ years; in the CIS group, it was $39.44 \pm 11.88$ years. The age comparison between the two groups was $\mathrm{t}=0.338$ with $\mathrm{p}=0.737$. The time to relapse in the MS group was 2-12 months in 19 cases, 13-24 months in seven cases, 25-36 months in five cases, and 58-116 months in three cases. The median time to relapse was 12 months, with an upper quartile $(\mathrm{Qu})$ of 23.7 months, and $91.2 \%$ of the patients relapsed within three years. The duration of follow-up in the CIS group was 37-48 months in nine cases, 49-60 months in nine cases, 61-72 months in four cases, and 85-113 months in three cases, with a median duration of 54.0 months. Of the 61 patients, $50.8 \%$ developed MS within three years, and of the 34 patients who developed MS, 55.9\% relapsed within one year.

\section{Clinical results}

In univariate analysis, the onset of 16 patients (47.1\%) in the MS group, and of 20 (74.1\%) in the CIS group, started with sensory symptoms. Patients with the onset of sensory symptoms were less likely to develop MS than were patients with an onset of motor dyskinesia (including an onset combined with sensation disorders) $(\mathrm{OR}=0.311)$. Sensory Functional
Table 1. Functional systems score for patients from clinically isolated syndrome (CIS) and multiple sclerosis (MS) groups at symptoms onset

\begin{tabular}{|c|c|c|c|}
\hline & \multirow[t]{2}{*}{ Score } & \multicolumn{2}{|c|}{ Number of patients } \\
\hline & & CIS & MS \\
\hline \multirow[t]{5}{*}{ Sensory FSS } & 0 & 0 & 2 \\
\hline & 1 & 1 & 0 \\
\hline & 2 & 18 & 17 \\
\hline & 3 & 5 & 11 \\
\hline & 4 & 3 & 4 \\
\hline \multirow[t]{6}{*}{ Pyramidal FSS } & 0 & 7 & 5 \\
\hline & 1 & 6 & 2 \\
\hline & 2 & 4 & 11 \\
\hline & 3 & 9 & 13 \\
\hline & 4 & 0 & 3 \\
\hline & 5 & 1 & 0 \\
\hline \multirow{5}{*}{$\begin{array}{l}\text { Bowel and } \\
\text { bladder FSS }\end{array}$} & 0 & 20 & 30 \\
\hline & 1 & 3 & 0 \\
\hline & 2 & 3 & 0 \\
\hline & 3 & 0 & 2 \\
\hline & 4 & 1 & 2 \\
\hline \multirow[t]{5}{*}{ Ambulation score } & 0 & 13 & 10 \\
\hline & 1 & 11 & 19 \\
\hline & 2 & 2 & 1 \\
\hline & 5 & 0 & 2 \\
\hline & 10 & 1 & 2 \\
\hline
\end{tabular}

FSS - Functional Systems Scores

Systems Scores (FSSs), pyramidal FSS, bowel and bladder FSS, and ambulation scores were analysed using a layer-by-layer single-factor analysis.

Only patients with a Kurtzke FSS of the pyramidal functions $\geq 2$ quickly progressed to MS (OR $=3.58)($ Tab. 1$)$. There was no significant difference in EDSS scores between the MS and CIS groups before (MS: median 3.5, minimum 2.0, maximum 7.0; CIS: median 3.0, minimum 2.0, maximum 7.0; $\mathrm{p}=0.143$ ) or after (MS: median 1.5, minimum 0 , maximum 4.5; CIS: median 2.0, minimum 0 , maximum $4.0 ; \mathrm{p}=0.985$ ) steroid treatment. The difference between the EDSS scores before and after steroid treatment was higher in the MS group (median 2.0, minimum 0, maximum 4.0) than in the CIS group (median 1.0, minimum 0, maximum 4.0) $(\mathrm{p}=0.001)$. There was a difference between the EDSS scores before and after steroid treatment with an EDSS of $\geq 1.5$ in 28 patients $(82.4 \%)$ in the MS group and nine patients (33.3\%) in the CIS group (OR = 9.333; Tab. 2).

Single-factor analysis was conducted for sensory, pyramidal, and bladder FSSs, as well as for ambulation score, respectively, in a layer-by-layer manner. Only patients with pyramidal FSS of $\geq 2$ were more likely to develop MS $(\chi 2=5.187 ; \mathrm{p}=0.023 ; \mathrm{OR}=3.582)$. 
Table 2. Comparison of clinical manifestations and results of auxiliary examinations between multiple sclerosis group and clinically isolated syndrome group

\begin{tabular}{|c|c|c|c|c|c|}
\hline & \multirow{2}{*}{$\begin{array}{l}\text { CIS group } \\
(n=27)\end{array}$} & \multirow{2}{*}{$\begin{array}{l}\text { MS group } \\
(n=34)\end{array}$} & \multirow[t]{2}{*}{ OR } & \multicolumn{2}{|c|}{$95 \% \mathrm{Cl}$} \\
\hline & & & & Lower limit & Upper limit \\
\hline First sensory symptoms & 20 & 16 & 0.311 & 0.104 & 0.928 \\
\hline FSS of pyramidal functions $\geq 2$ & 14 & 27 & 3.582 & 1.165 & 11.009 \\
\hline \multicolumn{6}{|l|}{ EDSS before and after steroid } \\
\hline$\geq 1.5$ & 9 & 28 & 9.333 & 2.838 & 30.700 \\
\hline$\geq 2.0$ & 4 & 19 & 7.283 & 2.068 & 25.657 \\
\hline Cerebrospinal fluid $\mathrm{OCB}+$ & 2 & 10 & 5.208 & 1.033 & 26.271 \\
\hline Increased 24-hour lgG synthetic rate in cerebrospinal fluid & 9 & 14 & 1.400 & 0.489 & 4.009 \\
\hline Increased lgG index in cerebrospinal fluid & 8 & 16 & 2.111 & 0.727 & 6.192 \\
\hline Spinal cord MRI Gadolinium enhancing lesion+ & 17 & 16 & 0.523 & 0.186 & 1.467 \\
\hline Spinal cord MRI lesion < 3 vertebral segments & 19 & 24 & 1.011 & 0.334 & 3.059 \\
\hline Multiple spinal cord MRI lesions & 2 & 3 & & & \\
\hline Cerebral MRI lesions meeting MAGNIMS & 0 & 2 & & & \\
\hline Abnormal VEP & 7 & 16 & 2.540 & 0.851 & 7.575 \\
\hline Abnormal BAEP & 6 & 6 & 0.750 & 0.212 & 2.658 \\
\hline Abnormal VEP + BAEP & 4 & 6 & 1.232 & 0.310 & 4.898 \\
\hline
\end{tabular}

BAEP — brainstem auditory evoked potentials; Cl — confidence interval; EDSS — Expanded Disability Status Scale; FSS — Functional Systems Scores; Ig — immunoglobulin G; MRI — magnetic resonance imaging; $\mathrm{OCB}$ - oligoclonal bands; $\mathrm{OR}$ — odds ratio; VEP — visual evoked potential

Table 3. Relationship between Expanded Disability Status Scale before and after steroid therapy and magnetic resonance imaging gadolinium enhancement in spinal cord lesions of CIS and MS groups

\begin{tabular}{|c|c|c|c|c|c|c|c|c|}
\hline & & \multirow{2}{*}{$\geq 1.5$} & \multirow{2}{*}{$<1.5$} & \multirow[t]{2}{*}{$x^{2}$} & \multirow[t]{2}{*}{$\mathbf{P}$} & \multirow[t]{2}{*}{ OR } & \multicolumn{2}{|c|}{$95 \% \mathrm{Cl}$} \\
\hline & & & & & & & $\begin{array}{l}\text { Lower } \\
\text { limit }\end{array}$ & $\begin{array}{c}\text { Upper } \\
\text { limit }\end{array}$ \\
\hline \multirow[t]{2}{*}{ CIS group } & Gd enhancement & 5 & 12 & 0.318 & 0.573 & 0.625 & 0.121 & 3.221 \\
\hline & No Gd enhancement & 4 & 6 & & & & & \\
\hline \multirow[t]{2}{*}{ MS group } & Gd enhancement & 13 & 3 & 0.250 & 0.874 & 0.867 & 0.148 & 5.059 \\
\hline & $\begin{array}{l}\text { No Gadolinium } \\
\text { enhancement }\end{array}$ & 15 & 3 & & & & & \\
\hline \multirow[t]{2}{*}{ Total } & Gadolinium enhancement & 18 & 15 & 1.125 & 0.289 & 0.568 & 0.199 & 1.621 \\
\hline & No Gadolinium enhancement & 19 & 9 & & & & & \\
\hline
\end{tabular}

\section{MRI results}

In the MS group, lesions were located in the cervical medulla in 19 cases (55.9\%), in the thoracic medulla in eight cases, in the cervicothoracic medulla in six cases, and in the thoracolumbar medulla in one case. There were 24 cases $(70.6 \%)$ with lesions of fewer than three vertebral segments, seven cases with lesions involving $4-9$ vertebral segments, and three cases with lesions involving more than 10 vertebral segments. Three cases had multiple spinal lesions, and 16 cases had lesion enhancements. Two cases showed a cerebral MRI that met the MRI in multiple sclerosis (MAGNIMS) criteria [1]; this was also the case for $>3$ periventricular lesions with enhancement (-) cases, who had relapsed seven and 58 months later, respectively.

In the CIS group, lesions were located in the cervical medulla in 15 cases (55.6\%), in the thoracic medulla in five cases, in the cervicothoracic medulla in six cases, and in the thoracolumbar medulla in one case. There were 19 cases $(70.4 \%)$ with lesions on fewer than three vertebral segments, six cases with lesions involving $4-9$ vertebral segments, and two cases with lesions involving more than 10 vertebral segments. Two cases had multiple spinal cord lesions, and 17 cases had Gd enhancing lesions. There were no cases with a cerebral MRI that met the MAGNIMS criteria. The differences in the spinal cord lesions involving $<3$ vertebral segments and Gd enhancing lesions were not statistically significant between the two groups. The relationship between spinal MRI Gd enhancing lesions and the EDSS before and after steroid treatment was further analysed (Tab. 3). The number of cases in the two groups was relatively small, and there was no significant difference between them. There was also no significant difference between the two groups in terms of cases with spinal cord Gd enhancing lesions. 


\section{Results of CSF and neurophysiological examinations}

There were 10 cases (29.4\%) in the MS group and two cases $(7.4 \%)$ in the CIS group with CFS-OCB $(+)(\mathrm{OR}=5.208)$ and serum OCB (-). The differences in the increased $24-\mathrm{h} \mathrm{IgG}$ synthetic rate in CSF, the increased IgG index in CSF, the abnormal VEP, and abnormal BAEPs were not statistically significant between the two groups (Tab. 2).

\section{Other results}

No patients showed abnormalities in the serum autoimmune antibody examination with AQP4-Ab (-) and MOG-Ab $(-)$ in either the serum or the CSF.

\section{Discussion}

Approximately $85 \%$ of patients with MS initially have CIS; approximately $45 \%$ of patients with CIS subsequently convert to clinically confirmed MS within two years, and spinal cord involvement is more common in patients with CIS [6-8].

In the present study, of the 61 patients who experienced a first episode of presenting with spinal CIS, 55.7\% converted to MS, $50.8 \%$ converted to MS within three years, and $55.9 \%$ of the 34 patients who converted to MS relapsed within one year. Differences in age and gender were not significant between the MS and CIS groups.

The clinical factors for CIS conversion to MS showed that patients with a polysymptomatic onset, and/or incomplete recovery from initial symptoms, reflected a higher risk of experiencing a second demyelinating attack. Motor symptoms at the onset may increase the probability of MS $[9,10]$. In the present study, univariate analysis showed that patients with pure sensory abnormalities at the onset were less likely to convert to MS (OR $=0.311)$, and those with an FSS of the pyramidal functions $\geq 2$ were more likely to advance to $\mathrm{MS}(\mathrm{OR}=3.582)$. After two weeks of steroid treatment, the median difference in EDSS between the two groups was 2 in the MS group and 1 in the CIS group, indicating a significant difference. Further analysis showed that there were fewer CIS patients with an EDSS difference $<1$ and $\geq 2.5$, which could not be analysed, and a difference of $\geq 1.5(\mathrm{OR}=9.333)$ and $\geq 2.0(\mathrm{OR}=7.283)$ indicated that MS was likely to progress. It has been reported that complete remission predicts a lower risk; the incomplete remission risk is 1.9 times higher [8]. Another study revealed that poor vs. complete recovery is predictive of a lower risk for another event within a year (hazard ratio $=0.48$ ). Perhaps patients with more concurrent or destructive demyelinating lesions are more prone to temporarily suppressing biological disease processes compared to those with a less aggressive disease onset [11]. The severity of disability calculated via EDSS was similar in MS and CIS patients. The EDSS scores of CIS patients decreased significantly faster than did those of MS patients on the first four days of steroid treatment. The EDSS scores of MS and CIS patients were recorded after five days of similar steroid treatment [12]. There was no significant difference in the EDSS scores between the CIS and MS groups after treatment in this study, suggesting that the neurological damage had been completely recovered and was not correlated with the conversion to MS. Further observation using a larger sample size is required.

Glucocorticoids have anti-inflammatory effects such as inducing lymphocyte apoptosis, reducing the expression of cell adhesion molecules, and inhibiting the synthesis and secretion of inflammatory cytokines and antibodies. Additionally, TBX21/Tbet, a Th1 cell-specific transcription factor, is selectively expressed in the TH1 cells and is significantly higher in CD4+ T-cells in patients with CIS who progress to MS compared to CIS patients who do not [13]. Soluble $\mathrm{CD} 27$ (sCD27) is a marker of intrathecal active T-cell-mediated inflammatory response. The levels of sCD27 were found to be significantly higher in patients who progressed to MS than in those who did not [14]. A study result showed that interferon gamma (IFN- $\gamma$ ) levels and the IFN- $\gamma /$ interleukin (IL)-10 ratio were higher in CIS patients than in relapsing-remitting (RR) MS patients. Th1 cell frequencies were higher in CIS and RRMS cases than in progressive MS cases, and RRMS had a higher Th17 frequency than CIS. The Th1/Th17 cell ratio was skewed toward Th1 in CIS compared to MS phenotypes [15]. A high central content for IL-8 was associated with the risk of progression to MS in subjects with CIS. Asymptomatic intrathecal inflammation placed subjects at risk for MS conversion, regardless of the lesion load. Moreover, CSF IL-8 levels were higher in RRMS cases with high disease activity. A higher number of relapses in the first two years after diagnosis and shorter first inter-attack intervals were observed in patients with high levels of IL-8 [16]. It was recently discovered that glucocorticoid-induced leucine zipper (GILZ) messenger ribonucleic acid (mRNA) levels were significantly lower on post-steroid treatment day five compared to day one in RRMS patients; there was no significant difference in CIS. Myeloid cell leukemia (MCL-1) mRNA levels were significantly lower on post-steroid treatment day five than the value measured on day one in the CIS group, but not in the RRMS group.

Glucocorticoid-induced leucine zipper is a glucocorticoid receptor (GR) transactivated target gene that exhibits potent anti-inflammatory properties by suppressing pro-inflammatory cytokines and via the modulation of T-cell action. This is thought to regulate immune response by modulating the T1/T2 balance, and it has been suggested that GILZ could represent an immunomodulatory target in autoimmune diseases. Furthermore, GILZ and MCL-1 play a crucial role in the regulation of GR-dependent apoptosis in lymphocytes [17]. Whether the reason for the better steroid therapeutic effects in the MS group (compared to the CIS group) correlated with this factor requires further investigation. However, clinicians should pay attention to EDSS differences before and after steroid treatment. 
The 2017 McDonald diagnostic criteria recommend that OCB-positive patients with CIS who meet the clinical or MRI spatial multiplicity criteria can be clinically diagnosed with MS [1]. Additionally, CFS-OCB-positive patients with CIS have a significantly increased risk of conversion to MS [18]. Oligoclonal bands are immunoglobulin produced in the sheath by $\mathrm{B}$ lymphocytes and plasma cells. Among the potential predictors of transition to MS in CIS patients, OCB-positive $\mathrm{OR}=5.985$ [3]. Predictors of conversion in multivariable analyses suggest that the hazard ratio of OCB-positive is 2.18 [7].

The results of the present study were consistent with the above criteria. However, the OCB positivity was only $19.7 \%$, which was consistent with a report compiled in Japan which reflected a positivity of $19.2 \%$ [18], but lower than the positivity of $68.6-82.4 \%$ reported by European studies [7, 19]. In the MS group, the OCB positivity was significantly higher than in the CIS group (OR $=5.208)$, suggesting that although the OCB positivity was low, OCB-positive patients were highly susceptible to converting to MS.

The MRI procedure is essential for diagnosing CNS demyelinating disease. The presence of lesions on an MRI, their location, number, and size are believed to be correlated with CIS conversion to MS. The risk of experiencing further demyelinating activity is strongly affected by the extent of the radiological inflammatory white matter damage on the baseline scan. The majority (72-89\%) of patients with abnormal brain imaging at the baseline are eventually diagnosed with MS. This proportion is even higher (98\%) when gadolinium-enhancing lesions are detected. A larger number of brain lesions on T2-weighted MRI sequences, and a more severe accumulation of lesions over the first five years from onset, proportionally increase the probability of developing MS [19-22]. In a United Kingdom cohort, over a 15-year mean follow-up period, MS developed among all patients with an abnormal spinal cord MRI at the baseline, but only in $58 \%$ of patients with no evidence of spinal cord inflammation [22]. Most spinal cord lesions in patients with MS include fewer than three vertebral segments; however, long spinal cord lesions are more common in Asian patients [1]. A study in Asian patients found that $16.3 \%$ presented with spinal cord lesions longer than three vertebral segments [23]. A differential diagnosis of MS and NMOSD and MOG antibody disease should be considered in patients with long spinal cord lesions.

In the present study, only two cases showed lesions on the cerebral MRI that met the MAGNIMS criteria, both of which progressed to MS, and only five cases had multiple spinal lesions, three of whom progressed to MS. The data could not be statistically analysed because of the small number of cases. Univariate analysis of the lesions that comprised fewer than three vertebral segments and lesion enhancement showed no statistically significant difference.

Therefore, the spinal cord lesions themselves may have no predictive significance in terms of imaging characteristics, and attention should be paid to the predictive significance of the cerebral MRI with lesions that meet the MAGNIMS criteria for the conversion of spinal CIS to MS.

In addition, OCB is more likely to be found among patients with abnormal baseline brain MRIs, and their predictive effect in the multivariate model remains significant, albeit modest [21]. According to the $2017 \mathrm{McD}$ onald diagnostic criteria for MS, patients who experienced a spinal cord injury for the first time presented lesions with their brain MRI results that conformed to the MAGNIMS criteria and were diagnosed as having MS with positive CSF-OCB. Compared to some cases in existing studies, the number of abnormal brain MRIs and positive OCB cases decreased. An analysis of 290 patients with a demyelinating disease found that $48 \%$ of CIS diagnoses were identified according to the $2010 \mathrm{McD}$ onald criteria. But with the application of the $2017 \mathrm{McD}$ onald criteria, only $6 \%$ of patients were diagnosed with CIS [24]. After changing the diagnostic criteria, it is necessary to conduct prospective observations with a larger sample size to observe whether the findings will be different from the original study results.

\section{Lack of impact of various spinal imaging parameters}

Both patients and physicians have overestimated the ability of modern imaging techniques to detect symptomatic spinal cord lesions and provide guidance for treatment. None of the imaging methods are highly sensitive or relatively specific. One of the major challenges of imaging examinations is determining the clinical relevance of spinal cord morphological abnormalities. In the absence of a clinical evaluation, imaging tests cannot determine whether a particular spinal cord structure reflects the cause of symptoms. Overemphasising imaging at the expense of relevant clinical symptoms is dangerous to both the patient and the physician, and can lead to inappropriate treatment.

An evidence-based medical study conducted by the Quality Standards Subcommittee of the American Academy of Neurology concluded that VEPs and SEPs may help identify patients at risk of developing MS [25]. Studies have also suggested that three abnormal EPs can help identify patients with CIS who are at a higher risk of disability, independent of the MRI results [26].

All cases in the present study were patients with spinal cord lesions, and not all were examined for SEPs. Univariate analysis of the VEPs and BAEPs showed no significant differences. Studies from Egypt and Germany suggest that VEP did not add further information concerning the conversion rate to MS $[27,28]$.

There are some limitations to the present study. Firstly, this was a retrospective case analysis and not a prospective study, making the risk of bias possible. Secondly, the present study was a single-centre clinical investigation. The sample size was small and, accordingly, it is necessary to increase this aspect and conduct multi-centre clinical studies. Eighty-five percent of MS cases begin with a group of symptoms called CIS, but 
$37 \%$ of CIS patients do not experience a relapse within 20 years of onset. Longer-term observations of patient outcomes are needed. Identifying biomarkers from biochemical, metabolic, and proteomic screens that predict clinically defined conversion from CIS to MS, as well as generating a multi-omics-based algorithm with higher prognostic accuracy, represents an important direction for future research [29].

The present study serves as a simple 'first step' in this process, and any potential predictors identified must be validated by a prospective study.

\section{Conclusions}

After the changes made to the $2017 \mathrm{McD}$ onald diagnostic criteria for MS, the number of patients diagnosed with CIS decreased; this underlines the need for continuous observation of outcomes for CIS patients. In patients with spinal CIS, those with pure sensory abnormalities at the onset were less likely to convert to MS $(\mathrm{OR}=0.311)$. The factors with higher risk were, in order, differences in EDSS before and after steroid therapy reflecting $\geq 1.5(\mathrm{OR}=9.333)$, positive CSF-OCB $(\mathrm{OR}=5.208)$, and those with an FSS of the pyramidal functions score $\geq 2(\mathrm{OR}=3.582)$. Attention should also be paid to patients with MRI lesions meeting the MAGNIMS criteria. There was no significant difference in nerve function recovery between CIS and MS patients after steroid therapy, but clinicians should pay attention to EDSS score reductions after steroid therapy compared to before treatment. Half of the patients converted to MS within three years, and half of those relapsed within one year. The $\mathrm{Qu}$ of the time to relapse was two years, suggesting it is necessary to focus clinical studies on patients susceptible to converting to MS within 1-2 years to facilitate early treatment with DMTs.

Ethics approval and consent to participate: This study was conducted with approval from the Ethics Committee of Peking University Third Hospital. This study was conducted in accordance with the Declaration of Helsinki, and written informed consent was obtained from all participants.

Conflict of interest: None.

Funding: None.

\section{References}

1. Thompson AJ, Banwell BL, Barkhof F, et al. Diagnosis of multiple sclerosis:2017 revisions of the McDonald criteria. Lancet Neurol. 2018;17(2): 162-173, doi: 10.1016/S1474-4422(17)30470-2, indexed in Pubmed: 29275977.

2. Miller D, Barkhof F, Montalban X, et al. Clinically isolated syndromessuggestive of multiple sclerosis, part I: natural history, pathogenesis, diagnosis, and prognosis. Lancet Neurol. 2005; 4(5): 281-288, doi: 10.1016/s1474-4422(05)70071-5, indexed in Pubmed: 15847841.

3. Kolčava J, Kočica J, Hulová M, et al. Conversion of clinically isolatedsyndrome to multiple sclerosis: a prospective study. Mult Scler
RelatDisord. 2020; 44: 102262, doi: 10.1016/j.msard.2020.102262, indexed in Pubmed: 32570179.

4. Izadi S, Ahmadi M, Poursadeghfard M, et al. Predictors and conversionrate of clinically isolated syndrome to clinically definite multiple sclerosis: a follow-up study in patients living in the southern part of Iran. Caspian Journal of Neurological Sciences. 2020; 6(1): 9-15, doi: 10.32598/cjns.6.20.200.3.

5. Andersson M, Alvarez-Cermeño J, Bernardi G, et al. Cerebrospinal fluid in the diagnosis of multiple sclerosis: a consensus report. J Neurol Neurosurg Psychiatry. 1994; 57(8): 897-902, doi: 10.1136/ jnnp.57.8.897, indexed in Pubmed: 8057110.

6. Miller D, Chard D, Ciccarelli O. Clinically isolated syndromes. LancetNeurol. 2012; 11(2): 157-169, doi: 10.1016/s1474-4422(11)70274-5, indexed in Pubmed: 22265211.

7. Kuhle J, Disanto G, Dobson R, et al. Conversion from clinically isolated syndrome to multiple sclerosis: A large multicentre study. Mult Scler. 2015; 21(8): 1013-1024, doi: 10.1177/1352458514568827, indexed in Pubmed: 25680984.

8. Eriksson M, Andersen O, Runmarker B. Long-term follow up of patients with clinically isolated syndromes, relapsing-remitting and secondary progressive multiple sclerosis. Mult Scler. 2003; 9(3): 260-274, doi:10.1191/1352458503ms914oa, indexed in Pubmed: 12814173.

9. Calabrese M, Marastoni D, Crescenzo F, et al. Early multiple sclerosis: diagnostic challenges in clinically and radiologically isolated syndrome patients. Curr Opin Neurol. 2021; 34(3): 277-285, doi: 10.1097/ WC0.0000000000000921, indexed in Pubmed: 33661162.

10. Grzegorski T, Losy J. What do we currently know about the clinically isolated syndrome suggestive of multiple sclerosis? An update. Rev Neurosci. 2020; 31(3): 335-349, doi: 10.1515/revneuro-2019-0084 ,indexed in Pubmed: 31811811.

11. Mowry EM, Pesic M, Grimes B, et al. Clinical predictors of early secondevent in patients with clinically isolated syndrome. J Neurol. 2009; 256(7): 1061-1066, doi: 10.1007/s00415-009-5063-0, indexed inPubmed: 19252775.

12. Piri Cinar B, Ozakbas S, Idiman E. Evaluation of clinical parameters during and after treatment of attack in patients with clinically isolated syndrome: comparison of the results with that of multiple sclerosis patients. Clin Neurol Neurosurg. 2013; 115(12): 2432-2437, doi:10.1016/j.clineuro.2013.09.003, indexed in Pubmed: 24140010.

13. Basdeo SA, Kelly S, O'Connell $\mathrm{K}$, et al. Increased expression of Tbet in $\mathrm{CD} 4(+) \mathrm{T}$ cells from clinically isolated syndrome patients at high risk of conversion to clinically definite MS. Springerplus. 2016; 5(1): 779, doi: 10.1186/s40064-016-2510-0, indexed in Pubmed: 27386265.

14. van der Vuurst de Vries RM, Mescheriakova JY, Runia TF, et al. SolubleCD27 levels in cerebrospinal fluid as a prognostic biomarker in clinically isolated syndrome. JAMA Neurol. 2017; 74(3): 286-292, doi:10.1001/jamaneurol.2016.4997, indexed in Pubmed: 28055081.

15. Arellano G, Acuña E, Reyes LI, et al. Th1 and Th17 cells and associated cytokines discriminate among clinically isolated syndrome and multiple sclerosis phenotypes. Front Immunol. 2017; 8: 753, doi: 10.3389/ fimmu.2017.00753, indexed in Pubmed: 28713377.

16. Rossi S, Motta C, Studer V, et al. Subclinical central inflammation is risk for RIS and CIS conversion to MS. Mult Scler. 2015; 21(11): 1443-1452, doi: $10.1177 / 1352458514564482$, indexed in Pubmed: 25583841.

17. Evangelopoulos ME, Nasiri-Ansari N, Kassi E, et al. Methylprednisolone stimulated gene expression (GILZ, MCL-1) and basal cortisol levels in multiple sclerosis patients in relapse are associated with clinical 
response. Sci Rep. 2021; 11(1): 19462, doi: 10.1038/s41598-02198868-y, indexed in Pubmed: 34593869.

18. Dobson R, Ramagopalan S, Davis A, et al. Cerebrospinal fluid oligoclonal bands in multiple sclerosis and clinically isolated syndromes: a meta-analysis of prevalence, prognosis and effect of latitude. J Neurol Neurosurg Psychiatry. 2013; 84(8): 909-914, doi: 10.1136/ jnnp-2012-304695, indexed in Pubmed: 23431079.

19. Gabelić T, Radmilović M, Posavec V, et al. Differences in oligoclonal bands and visual evoked potentials in patients with radiologically andclinically isolated syndrome. Acta Neurol Belg. 2013; 113(1): 13-17, doi: 10.1007/s13760-012-0106-1, indexed in Pubmed: 22740024.

20. Chung KK, Altmann D, Barkhof F, et al. A 30-year clinical and magneticresonance imaging observational study of multiple sclerosis and clinically isolated syndromes. Ann Neurol. 2020; 87(1): 63-74, doi:10.1002/ana.25637, indexed in Pubmed: 31693200.

21. Tintore M, Rovira À, Río J, et al. Defining high, medium and lowimpact prognostic factors for developing multiple sclerosis. Brain.2015; 138(Pt 7): 1863-1874, doi: 10.1093/brain/awv105, indexed in Pubmed: 25902415.

22. Brownlee WJ, Altmann DR, Prados F, et al. Early imaging predictorsof long-term outcomes in relapse-onset multiple sclerosis. Brain. 2019; 142(8): 2276-2287, doi: 10.1093/brain/awz156, indexed in Pubmed: 31342055.

23. Wang KC, Lin KH, Lee TC, et al. Poor responses to interferon-beta treatment in patients with neuromyelitis optica and multiple sclerosis with long spinal cord lesions. PLoS One. 2014; 9(6): e98192, doi: 10.1371/journal.pone.0098192, indexed in Pubmed: 24887452.
24. Lee DH, Peschke M, Utz KS, et al. Diagnostic value of the 2017 McDonald criteria in patients with a first demyelinating event suggestive of relapsing-remitting multiple sclerosis. Eur J Neurol. 2019; 26(3):540545, doi: 10.1111/ene.13853, indexed in Pubmed: 30362206.

25. Gronseth GS, Ashman EJ. Practice parameter: the usefulness of evoked potentials in identifying clinically silent lesions in patients with suspected multiple sclerosis (an evidence-based review): Report of the Quality Standards Subcommittee of the American Academy of Neurology. Neurology. 2000; 54(9): 1720-1725, doi: 10.1212/ wnl.54.9.1720, indexed in Pubmed: 10802774.

26. Pelayo R, Montalban X, Minoves T, et al. Do multimodal evoked potentialsadd information to MRI in clinically isolated syndromes? MultScler. 2010; 16(1): 55-61, doi: 10.1177/1352458509352666, indexed in Pubmed: 19995838.

27. Shaheen H, Sayed S, Daker L, et al. Early predictors of conversion in patients with clinically isolated syndrome: a preliminary Egyptianstudy. Egyptian J Neurol Psych Neurosurg. 2018; 54(1), doi: 10.1186/ s41983-018-0021-3.

28. Schwenkenbecher P, Sarikidi A, Bönig L, et al. Clinically isolated syndrome according to mcdonald 2010: intrathecal igg synthesis still predictivefor conversion to multiple sclerosis. Int J Mol Sci. 2017; 18(10):2061, doi: 10.3390/ijms18102061, indexed in Pubmed: 28953254.

29. Probert F, Yeo T, Zhou Y, et al. Integrative biochemical, proteomics and metabolomics cerebrospinal fluid biomarkers predict clinical conversion to multiple sclerosis. Brain Commun. 2021; 3(2): fcab084, doi: 10.1093/braincomms/fcab084, indexed in Pubmed:33997784. 